\title{
PROLAPSO UTERINO EM GATA - RELATO DE CASO
}

\author{
Rallyson Ramon Fernando Barbosa Lopes ${ }^{1 *}$ \\ Ana Maria Quessada ${ }^{2}$ \\ Ciro José Sousa de Carvalho ${ }^{3}$ \\ Jaknea dos Santos Leal ${ }^{4}$ \\ Karoline Gonçalves Moraes ${ }^{5}$ \\ Kennya Cristina Damasceno de Jesus ${ }^{6}$ \\ Talita Bianchin Borges ${ }^{7}$
}

LOPES, R. R. F. B.; QUESSADA, A. M.; CARVALHO, C. J. S. de; LEAL, J. dos S.; MORAES, K. G.; JESUS, K. C. D. de; BORGES, T. B. Prolapso uterino em gata - relato de caso. Arq. Ciênc. Vet. Zool. UNIPAR, Umuarama, v. 18, n. 2, p. 133-135, abr./jun. 2015.

RESUMO: O prolapso uterino em cadelas e gatas é uma emergência reprodutiva na qual ocorre eversão de um ou ambos os cornos uterinos através da vagina, durante ou após o parto. Tal patologia não é frequente em gatas, por isso o presente relato tem o objetivo de descrever um caso de prolapso uterino em uma gata atendida no Hospital Veterinário da Universidade Federal do Piauí. Na anamnese o proprietário se queixou de massa avermelhada na vulva após a expulsão de dois filhotes. Ao exame clínico, observou-se que a massa era o útero prolapsado. $\mathrm{O}$ animal foi encaminhado imediatamente ao centro cirúrgico para redução do útero prolapsado e realização de ovariosalpingohisterectomia (OSH). Após a OSH, o animal permaneceu internado durante 24 horas. Findo este período o animal teve alta e apresentou boa recuperação.

PALAVRAS-CHAVE: Felino.Útero.Ectopia. Eversão uterina.

\section{UTERINE PROLAPSE IN A CAT - A CASE REPORT}

\begin{abstract}
Uterine prolapse in dogs and cats is a reproductive emergency in which there is the protrusion of one or both uterine horns through the vagina during or after delivery. This condition is not common in cats, so this report aims to describe a case of uterine prolapse in a cat treated at the Veterinary Hospital of the Federal University of Piaui. Upon anamnesis, the owner complained of a reddish mass in the vulva after the expelling of two kittens. Clinical examination revealed that the mass was the prolapsed uterus. The animal was immediately sent to the operating room for reduction of the prolapsed uterus and an ovariosalpingohisterectomy $(\mathrm{OSH})$ was performed. After OSH, the animal remained hospitalized for 24 hours. After this period, the animal was discharged and recovered well.
\end{abstract}

KEYWORDS: Feline. Uterus. Ectopy. Uterine eversion.

\section{PROLAPSO UTERINO EN GATA - RELATO DE CASO}

RESUMEN: El prolapso uterino en perras y gatas es una emergencia reproductiva en la cual ocurre eversión de uno o ambos los cuernos uterinos a través de la vagina, durante o después del parto. Tal patología no es común en gatas, este informe tiene como objetivo describir un caso de prolapso uterino en una gata tratada en el Hospital Veterinario de la Universidad Federal de Piauí. En la anamnesis el propietario se quejó de una masa rojiza en la vulva después de la expulsión de dos gatitos. El examen clínico reveló que la masa era el prolapso de útero. El animal fue enviado de inmediato al centro quirúrgico para reducción del prolapso de útero y realización de ovariosalpinghisterectomía (OSH). Después de realizada la OSH, el animal permaneció hospitalizado durante 24 horas. Después de este período, el animal fue dado de alta y se recuperó bien.

PALABRAS CLAVE: Felino. Útero. Ectopia. Eversión uterina.

\section{Introdução}

O prolapso uterino é uma emergência em que ocorre eversão e protrusão de uma porção do útero através da cérvix na vagina durante ou próximo ao parto e não é frequente em cadelas e gatas (HEDLUND, 2008). Em gatas podem aparecer em animais com idade entre dois e seis anos que pariram duas ou mais vezes sem dificuldade. Usualmente esta enfermidade ocorre durante o parto prolongado ou após este (48 horas), quando a cérvix ainda se encontra dilatada (OZYURTLU; KAYA, 2005) sendo que um (NÖTHLING et al., 2002) ou ambos cornos uterinos podem prolapsar (MOSTACHIO et al., 2008; ROCHA et al., 2010). São fatores predisponentes para a enfermidade: relaxamento da musculatura pélvica, atonia uterina, separação incompleta das membranas placentárias, flacidez mesovariana, tenesmo e contrações uterinas excessivas (NÖTHLING et al., 2002; LEAL; PRESTES; BICUDO, 2003).

DOI: https://doi.org/10.25110/arqvet.v18i2.2015.5385

${ }^{1}$ Médico Veterinário, Clínica Criar Centro Veterinário, Rua Anfrísio Lobão, Jokey, Teresina- PI.*Autor para correspondência. E-mail: rallyson.medvet@ gmail.com

${ }^{2}$ Professora Titular Doutora, Mestrado em Ciência Animal, UNIPAR, Praça Mascarenhas de Moraes, 4282, Zona III, Umuarama, PR.

${ }^{3}$ Pós graduação em Ciência Animal, Universidade Federal do Piauí(UFPI), Teresina- PI.

${ }^{4}$ Pós-graduação em Ciências Veterinárias no Semiárido-UNIVASF, Campus CiênciasAgrárias, Rodovia BR 407, Km 12, Petrolina-PE.

${ }^{5}$ Pós-graduação em Ciência Animal, Universidade Estadual do Maranhão (UEMA), São Luís- MA.

${ }^{6}$ Residência em Clínica e Cirurgia de Cães e Gatos da Universidade Federal do Piauí (UFPI), Teresina, PI.

${ }^{7}$ Pós-graduação em Ciência Animal, Bolsista PIT, Universidade Paranaense (UNIPAR), Praça Mascarenhas de Moraes, 4282, Zona III, Umuarama, PR. 
Esta patologia pode resultar em laceração do ligamento largo do útero e hemorragia da artéria uterina. A hemorragia pode ocasionar choque hipovolêmico, a menos que ela seja controlada rapidamente (HEDLUND, 2008). Em alguns casos, a vesícula urinária pode estar envolvida no prolapso (ROCHA et al., 2010).

O diagnóstico clínico deve ser baseado nos sinais, idade, fase do ciclo estral e confirmado pelo exame clínico e citologia vaginal (PINTO FILHO; HENRIQUES; DALMOLIN, 2002).

A terapia depende do resultado do exame físico e da viabilidade da mucosa uterina. Em prolapsos recentes, onde o dano do tecido uterino é mínimo, pode-se proceder à reposição do útero e cornos uterinos manualmente para a cavidade abdominal. A mucosa uterina deve ser limpa com solução salina e lubrificada antes deste procedimento (JOHNSTON; KUSTRITZ; OLSON, 2002).

O tratamento cirúrgico consiste na reposição do útero e evitar infecções. Outros tratamentos incluem: redução manual do prolapso por meio de laparotomia, redução manual do prolapso seguido de ovariosalpingohisterectomia $(\mathrm{OSH})$ e amputação do útero evertido (LEAL; PRESTES; BICUDO, 2003; OZYURTLU; KAYA, 2005; ROCHA et al., 2010).

O prognóstico está na dependência do grau e tempo de ocorrência (TONIOLLO; VICENTE, 2003). O prognóstico após a castração é excelente, caso se trate apropriadamente o choque e a hemorragia no pré-operatório (HEDLUND, 2008).

Resolveu-se relatar o presente caso porque o prolapso uterino em gatas é raro (LEAL; PRESTES; BICUDO, 2003).

\section{Relato do Caso}

Foi atendida no Hospital Veterinário de Teresina Piauí, uma gata SRD de três anos, pesando $3,5 \mathrm{Kg}$, em trabalho de parto. Segundo o proprietário, o felino tivera dois filhotes e, cerca de 24 horas após o parto, foi observada uma massa de aspecto avermelhado na região da vulva(Figura 1).

Figura 1: Felino, SRD,três anos de idade, apresentando massa de coloração avermelhada na região vulvar.

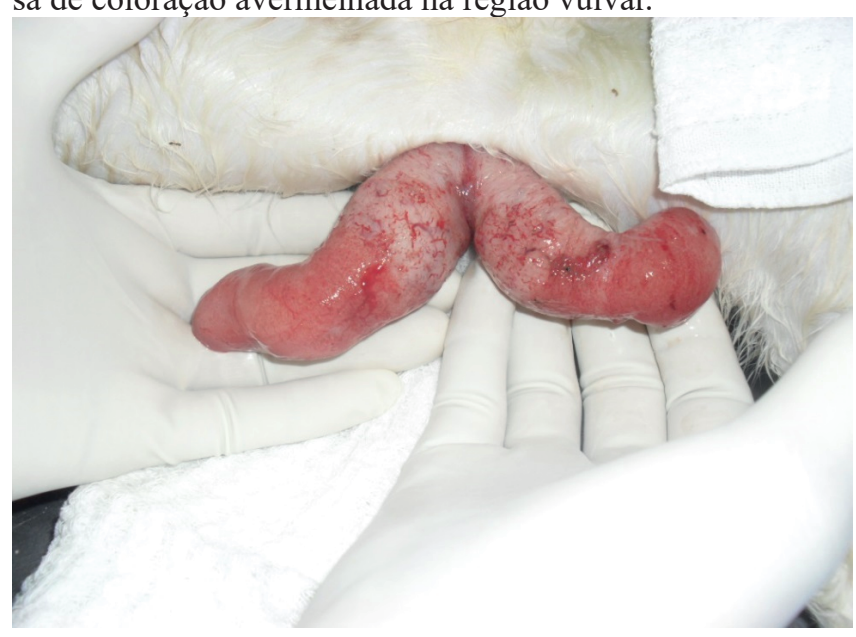

Ao exame clínico, o animal apresentava estado nutricional bom, temperatura normal $\left(38,2^{\circ} \mathrm{C}\right)$, mucosas normo- coradas e prolapso total do útero. Realizou-se coleta de sangue para hemograma e bioquímico, sendo que os resultados foram normais para a espécie.

Após diagnóstico, o animal foi encaminhado ao centro cirúrgico para redução do prolapso e posterior realização de OSH.

Como medicação pré-anestésica utilizou-se acepromazina $^{1}(0,1 \mathrm{mg} / \mathrm{kg})$ e tramadol ${ }^{8}(2 \mathrm{mg} / \mathrm{kg})$. Após 15 minutos, induziu-se a anestesia com cetamina ${ }^{9}(3 \mathrm{mg} / \mathrm{kg})$ e midazolan ${ }^{10}$ $(0,4 \mathrm{mg} / \mathrm{kg})$. A manutenção anestésica foi feita por inalação de isoflurano ${ }^{11}$. O útero prolapsado foi lavado com solução fisiológica ${ }^{12}$, em seguida aplicou-se solução hiperósmotica para redução do volume e o útero foi reintroduzido para a cavidade abdominal.

A seguir, foi realizada OSH por técnica tradicional. Após a cirurgia, animal foi internado e foi dada alta 24 horas após o procedimento cirúrgico. Nesta ocasião foram prescritos para o pós-operatório, cefalexina ${ }^{13}(20 \mathrm{mg} / \mathrm{kg})$, tramadol $^{2}$ $(2 \mathrm{mg} / \mathrm{kg})$, limpeza da ferida e aplicação de pomada cicatrizante $^{14}$ por 10 dias.

\section{Discussão}

Prolapso uterino em gatas não são frequentes (LEAL; PRESTES; BICUDO, 2003), sendo que no intervalo entre 1961 a 2008 (47 anos) foram resgatados na literatura consultada apenas nove relatos deste tipo de patologia na espécie, ou seja, menos de 0,2 casos por ano (NÖTHLING et al., 2002; ÖZYURTLU; KAYA, 2005), demonstrando que a enfermidade em felinos é rara. No Brasil, o primeiro caso publicado na literatura foi no ano de 1992 (SOARES; SUZUKI, 1992) e o último relato foi constatado em 2008 (MOSTACHIO et al, 2008). Um dos motivos da raridade da patologia em felinos pode estar relacionado ao fato de que tal espécie ainda é pouco encontrada nos lares brasileiros (DIAS et al., 2004). Além disso, a procura por castração de gatas é mais frequente do que a castração de cadelas (CARVALHO et al., 2007).

A idade da gata, o período de aparecimento (puerpério) e os aspectos clínicos gerais da enfermidade foram semelhantes aos descritos na literatura (ÖZYURTLU; KAYA, 2005; MOSTACHIO et al., 2008). No caso ora descrito o prolapso foi total, ou seja, abrangeu os dois cornos, como foi observado em outro relato (MOSTACHIO et al., 2008).

Por se tratar de uma emergência (HEDLUND, 2008), inclusive com possibilidade de óbito (MOSTACHIO et al., 2008), a gata foi imediatamente encaminhada ao centro cirúrgico.

A reintrodução do prolapso, seguida de OSH é um dos tratamentos preconizados (OZYURTLU; KAYA, 2005; MOSTACHIO et al., 2008; ROCHA et al., 2010), principalmente quando não há interesse em se conservar o animal para reprodução como ocorreu neste caso. $\mathrm{O}$ animal se recuperou bem, confirmando o prognóstico excelente da patologia (HE-

\footnotetext{
${ }^{8}$ Acepromazina,Syntec, Cotia- SP

Tramadol, Hipolabor, Sabará - MG.

${ }^{9}$ Cetamina, Syntec, Cotia - SP.

${ }^{10}$ Midazolam, Hipolabor, Sabará - MG.

${ }^{11}$ Isofluorano, Cristália, Itapira - SP.

${ }^{12}$ Solução fisiológica, Farmace, Barbalha - CE.

${ }^{13}$ Cefalexina ABL antibiótico do Brasil.

${ }^{14}$ Vetaglós, Vetnil, Louveira, SP.
} 
DLUND, 2008).

\section{Conclusão}

O prolapso uterino em gata tem bom prognóstico se houver atendimento imediato. Se não houver interesse em se conservar o animal para reprodução o melhor tratamento é a OSH.

\section{Referências}

CARVALHO, M. P. P. et al. Estudo retrospectivo da esterilização em cães e gatos no município de Araçatuba. Revista Ciência em Extensão, São Paulo, v. 3, n. 2, p. 81, 2007.

DIAS, R. A. et al. Estimativa de populações canina e felina domiciliadas em zona urbana do Estado de São Paulo.

Revista de Saúde Pública, São Paulo, v. 38, n. 4, p. 565$570,2004$.

HEDLUND, C. S. Cirurgia dos sistemas reprodutivo e genital. In: FOSSUM, T. W. Cirurgia de pequenos animais. 3. ed. Rio de Janeiro: Elsevier, 2008. p. 702-774.

JOHNSTON, S. D.; KUSTRITZ, M.V. R.; OLSON, P. N. $\mathrm{S}$. Canine and feline theriogenology. Philadelphia: W. B. Saunders, 2002. 592 p.

LEAL, L. S. et al. Prolapso uterino em gata - relato de três casos. Clínica Veterinária, São Paulo, v. 8, n. 46, p. 56-58, 2003

MOSTACHIO, G. Q. et al. Prolapso uterino em gata e retroflexão uterina em cadela. Ciência Animal Brasileira, Goiânia, v. 3, p. 801-805, 2008.

NÖTHLING, J. O. et al. Uterine prolapse with an interesting vascular anomaly in a cheetah: a case report. Theriogenology, Philadelphia, v. 58, p. 1705-1712, 2002.

ÖZYURTLU, N.; KAYA, D. Unilateral uterine prolapse in a cat. Turkish Journal of Veterinary and Animal Sciences, Ankara, v. 29, p. 941-943, 2005.

PINTO FILHO, S. T. L.; HENRIQUES, G. B.;

DALMOLIN, F. Hiperplasia e prolapso vaginal em cadelas - relato de caso. Revista da FVZA, Uruguaiana, v. 9, p. 24-28, 2002.

ROCHA, L. B. et al. Prolapso uterino em cadela com vesícula urinária encarcerada. Medicina Veterinária, Recife, v. 4, n. 2, p. 16-21, 2010.

SOARES, J. A. G.; SUZUKI, L. M. Prolapso uterino em felinos: relato de um caso. Semina: Ciências Agrárias, Londrina, v. 13, n. 1, p. 78-79, 1992.

TONIOLlO, G. H.; VICENTE, W. R. R. Manual de obstetrícia veterinária. São Paulo: Varela, 2003. 102 p.
Recebido em: 05.08.2014 Aceito em: 04.08.2015 